\title{
First $\beta$-beating measurement and optics analysis for the CERN Large Hadron Collider
}

\author{
M. Aiba, S. Fartoukh, A. Franchi, M. Giovannozzi, V. Kain, M. Lamont, R. Tomás, ${ }^{*}$ G. Vanbavinckhove, \\ J. Wenninger, and F. Zimmermann \\ CERN, CH 1211 Geneva 23, Switzerland \\ R. Calaga \\ BNL, Upton, New York 11973, USA
}

A. Morita

KEK, Tsukuba, Ibaraki 305-0801, Japan

(Received 15 June 2009; published 13 August 2009)

\begin{abstract}
Proton beams were successfully steered through the entire ring of the CERN Large Hadron Collider (LHC) on September the 10th of 2008. A reasonable lifetime was achieved for the counterclockwise beam, namely beam 2 , after the radiofrequency capture of the particle bunch was established. This provided the unique opportunity of acquiring turn-by-turn betatron oscillations for a maximum of 90 turns right at injection. Transverse coupling was not corrected and chromaticity was estimated to be large. Despite this largely constrained scenario, reliable optics measurements have been accomplished. These measurements together with the application of new algorithms for the reconstruction of optics errors have led to the identification of a dominant error source.
\end{abstract}

DOI: 10.1103/PhysRevSTAB.12.081002

PACS numbers: 41.85.-p, 29.20.dk, 29.27.Eg

\section{INTRODUCTION}

The LHC has a beta-beating tolerance lower than any other previous hadron collider. Table I shows the LHC beta-beating tolerances from Ref. [1], as derived from its tight mechanical aperture. To achieve the required control of the beta beating, the use of the most accurate numerical algorithms, as well as a highly performing beam position monitor (BPM) system, is mandatory. After dedicated studies spanning over several years, the procedures to measure and correct the optics of the LHC ring have been established via numerical simulations and measurements in existing accelerators [2-5]. In parallel, a complete and accurate magnetic model of the LHC has been developed based on measured field and alignment errors [6,7]. These studies show expected beta-beating levels at injection close to the above tolerances. Higher-order nonlinear magnetic errors can generate quadrupolar errors via feeddown. The specifications given in Ref. [1] aimed at providing beam-dynamical driven bounds on the components $b_{n}$, $a_{n}$ of the multipolar expansions of the magnetic field. The definition of the components $b_{n}, a_{n}$ is also given in Ref. [1].

On 10 September 2008 the first beams were successfully injected and circulated for multiple revolutions along the LHC ring. This was the result of a meticulous preparation as well as several synchronization tests performed during the summer of 2008 [8]. In particular, following the achievements of September 10th, the counterclockwise

*rogelio.tomas@cern.ch beam was circulated in the LHC with an excellent lifetime on September 12th, after rf capture was established [9]. A typical beam consisted of a single bunch of about $2 \times 10^{9}$ protons, with transverse rms geometric emittances of $5.6 \times$ $10^{-9} \mathrm{~m}$ and $2.3 \times 10^{-9} \mathrm{~m}$ in the horizontal and vertical planes, respectively, and as measured in the transfer line [10]. The rms bunch length was about $0.5 \mathrm{~ns}$. At this stage of the beam commissioning it was possible to acquire the turn-by-turn beam positions over the first 90 turns at about 500 double-plane BPMs using the YASP [11] software. At this commissioning stage the BPM system was set up to acquire 90 turns, but it was planned to increase this number at a later stage. The ring rms horizontal and vertical closed orbits were corrected to 1.3 and $2.1 \mathrm{~mm}$, respectively. The injection oscillations enabled measuring optics parameters by means of the techniques developed in previous years to extract the maximum information from the available data set.

TABLE I. Relative peak beta-beating tolerances for the LHC as extracted from Ref. [1]. The off-momentum tolerances are given at the maximum momentum deviation of $\delta_{p}=1.5 \times 10^{-3}$ and $\delta_{p}=0.86 \times 10^{-3}$ for injection and collision optics, respectively.

\begin{tabular}{lcccc}
\hline \hline & \multicolumn{2}{c}{ On-momentum } & \multicolumn{2}{c}{ Off-momentum } \\
& $\left(\frac{\Delta \beta_{x}}{\beta_{x}}\right)_{\text {peak }}$ & $\left(\frac{\Delta \beta_{y}}{\beta_{y}}\right)_{\text {peak }}$ & $\left(\frac{\Delta \beta_{x}}{\beta_{x}}\right)_{\text {peak }}$ & $\left(\frac{\Delta \beta_{y}}{\beta_{y}}\right)_{\text {peak }}$ \\
\hline Injection optics & $14 \%$ & $16 \%$ & $7 \%$ & $5 \%$ \\
Collision optics & $15 \%$ & $19 \%$ & $10 \%$ & $6 \%$ \\
\hline \hline
\end{tabular}


In this paper the different techniques used to measure the machine optics are presented in Sec. II. Section III describes new optics error reconstruction algorithms which have been developed and applied to this exceptional situation with very limited data plus the unavailability of the machine to iterate corrections. The coupling measurement from the secondary spectral lines of BPM data is presented in Sec. IV. Finally, an error analysis based on numerical simulations is given in the Appendix.

\section{THE BETA-BEATING MEASUREMENT}

The LHC beam 2 optics measurement was severely limited due to the availability of only a single turn-byturn BPM data acquisition right at injection and containing only 90 turns. In addition the transverse coupling was uncorrected and the chromaticity was estimated to be 30 units [12] based on the synchrotron sidebands of the transverse spectrum. Despite these setbacks, reliable optics measurements were accomplished [13].

The optics is probed through the phase advance between BPMs as it provides a robust and calibration-independent observable. The beta functions are extracted from the phase advances between three BPMs as it was done in the Large Electron Positron collider (LEP) [14]. No statistical error can be assigned to the measurement due to the existence of only a single data acquisition. However, by using multiple combinations of three BPMs, various beta functions measurements can be obtained at the same BPM. The average and rms of these measurements yield the beta function and its error bar, respectively. Prior to this analysis faulty BPMs are identified and discarded following various approaches $[15,16]$. The robustness of this measurement is assessed in the Appendix by simulating the measurement with the best knowledge of the machine conditions and the instrumentation. The simulations reveal that the measured horizontal and vertical beta functions differ from the input model values by $5 \%$ and $3 \% \mathrm{rms}$, respectively.

Three different algorithms are used to measure the phase advance between BPMs, namely singular value decomposition (SVD) [17,18], Sussix [19], and harmonic analysis (or discrete-time Fourier transform). The three algorithms yield consistent beta function measurements as shown in Fig. 1. However, the SVD approach features a more accurate measurement as displayed in the histograms of the beta functions relative error (Fig. 2). The vertical beta function is measured with an rms error of about $2 \%$, while for the horizontal plane the rms error is $4 \%$. In [3] the following relation between rms phase beating and beta beating was obtained via simulations for the LHC:

$$
\Delta \phi_{\mathrm{rms}}=\frac{1}{\sqrt{2}}\left(\frac{\Delta \beta}{\beta}\right)_{\mathrm{rms}},
$$

which is also analytically expected since the phase advance between the LHC arc BPMs is 45 degrees. According to this relation, the measured rms beta-beating errors corre-

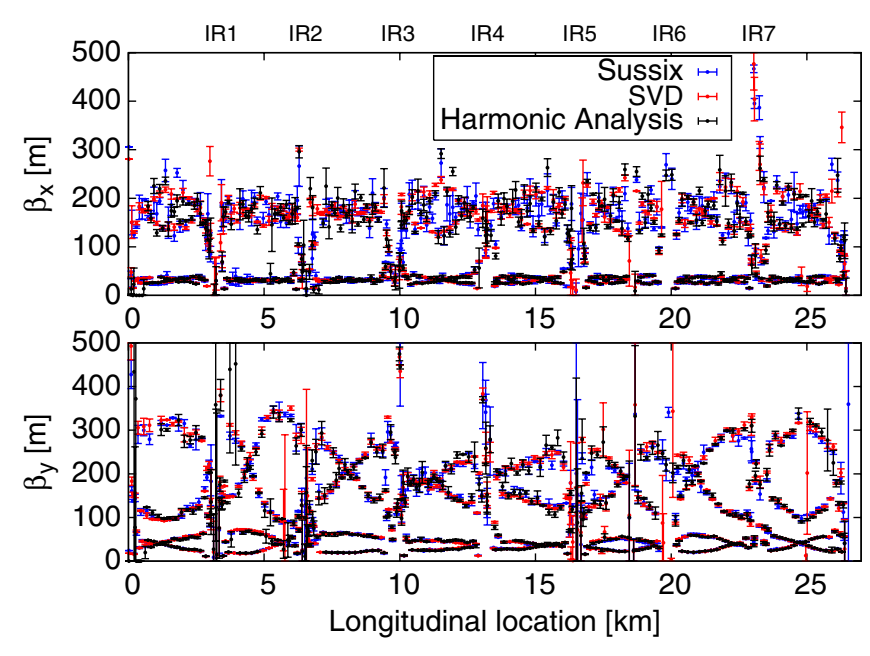

FIG. 1. (Color) The measurement of the horizontal (top) and vertical (bottom) $\beta$ functions for the LHC beam 2. The three different algorithms show consistent results. The locations of the LHC interaction regions (IRs) are marked on the top.

spond to vertical and horizontal rms phase errors of 0.8 and 1.6 degrees, respectively. The vertical error is already below the $1^{\circ}$ resolution, which is the target of the LHC optics measurements. At $1^{\circ}$ resolution Monte Carlo simulations of LHC optics corrections show a few percent probability of failure to achieve optics specifications [4]. The better performance of the SVD algorithm can be attributed to the fact that it takes advantage of the correlation between a large set of BPMs as in the case of the LHC. For these reasons, the SVD measurement is used as the reference in the rest of the paper. Alpha functions are
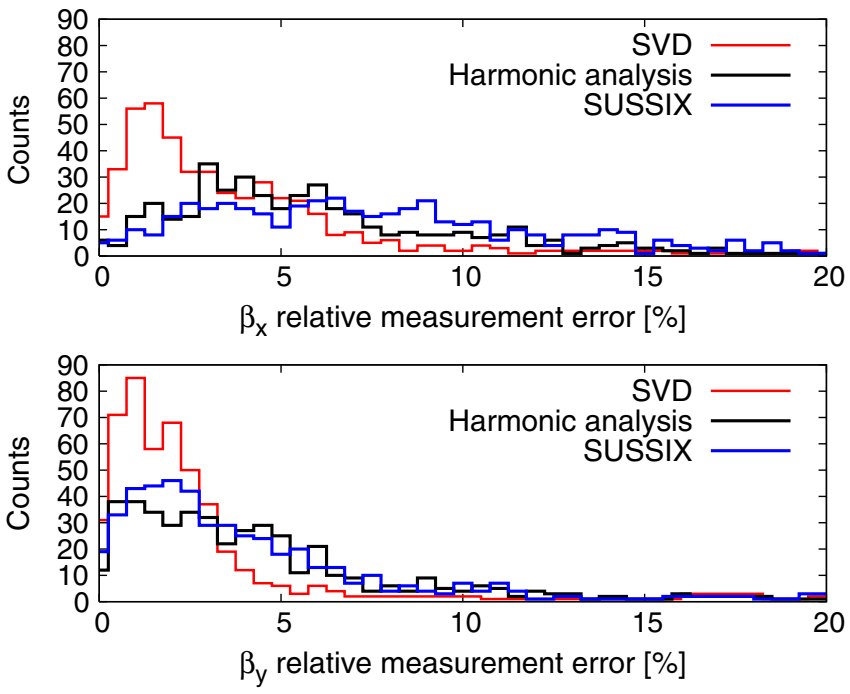

FIG. 2. (Color) Comparison of the resulting measurement errors for the three algorithms used to reconstruct the $\beta$ functions. Histograms of the horizontal and vertical beta function relative measurement errors are shown on the top and bottom plots, respectively. 

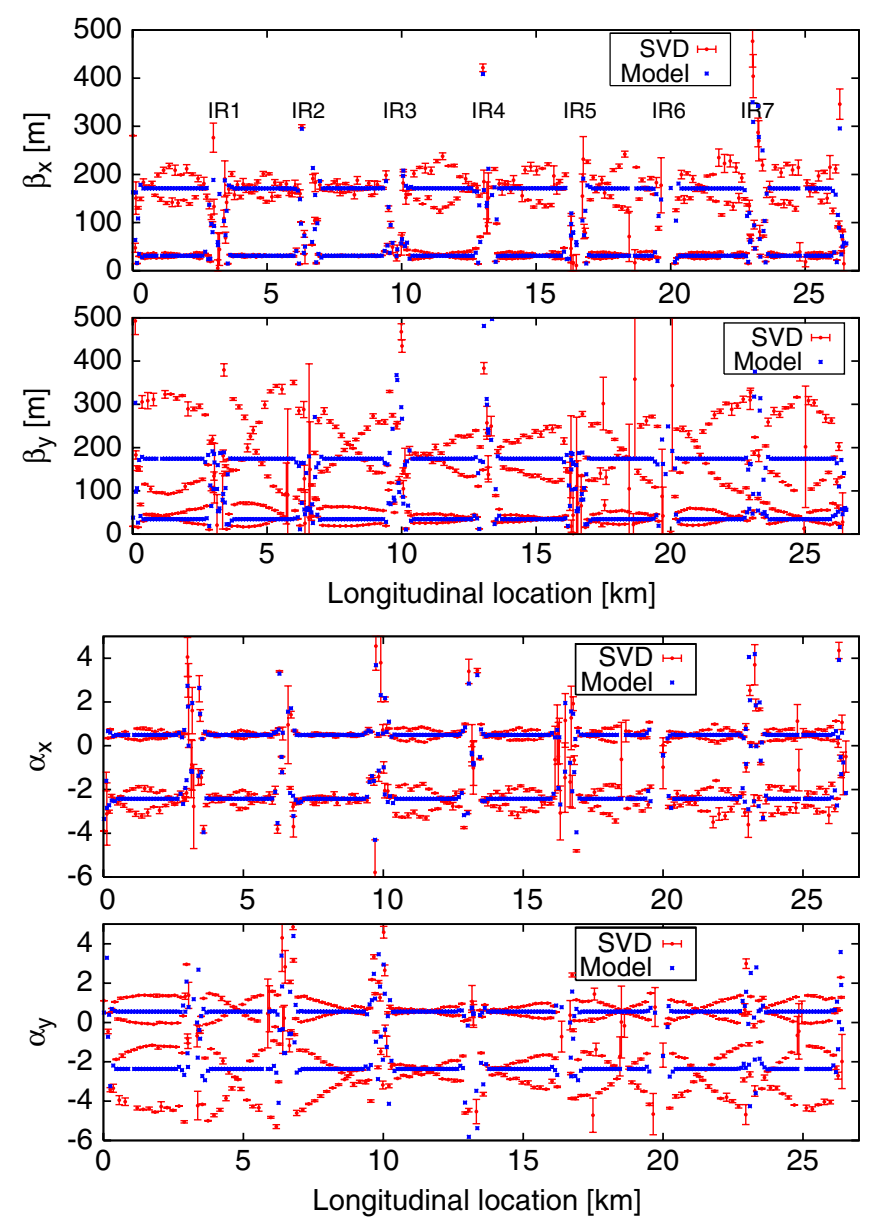

FIG. 3. (Color) Measured $\beta$ functions (top two plots) and $\alpha$ functions (bottom two plots) for the LHC beam 2 together with the design values.

measured in the same way as beta functions. Figure 3 shows the SVD based beta and alpha measurements together with the ideal model. Although $\alpha_{x, y}$ contain the
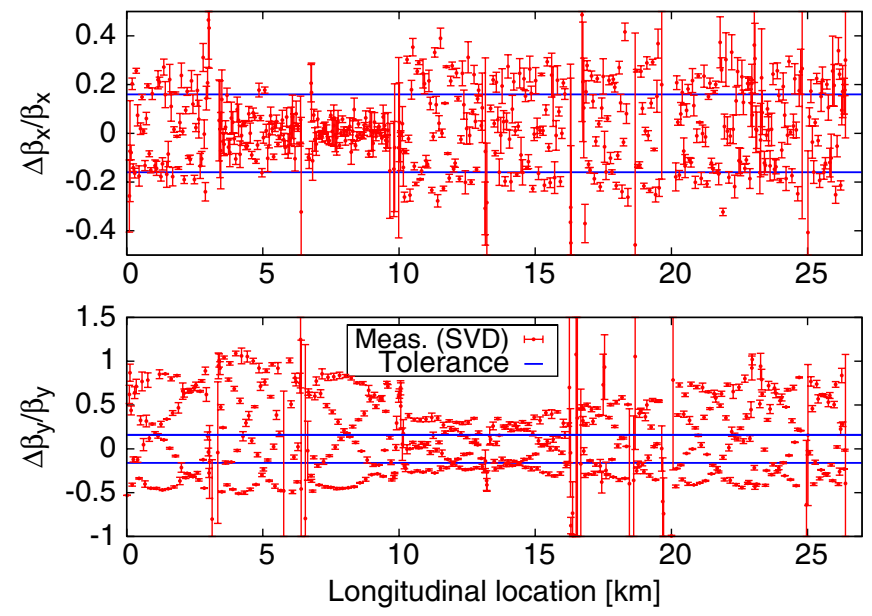

FIG. 4. (Color) Measured horizontal (top) and vertical (bottom) beta beating versus longitudinal location together with tolerances.

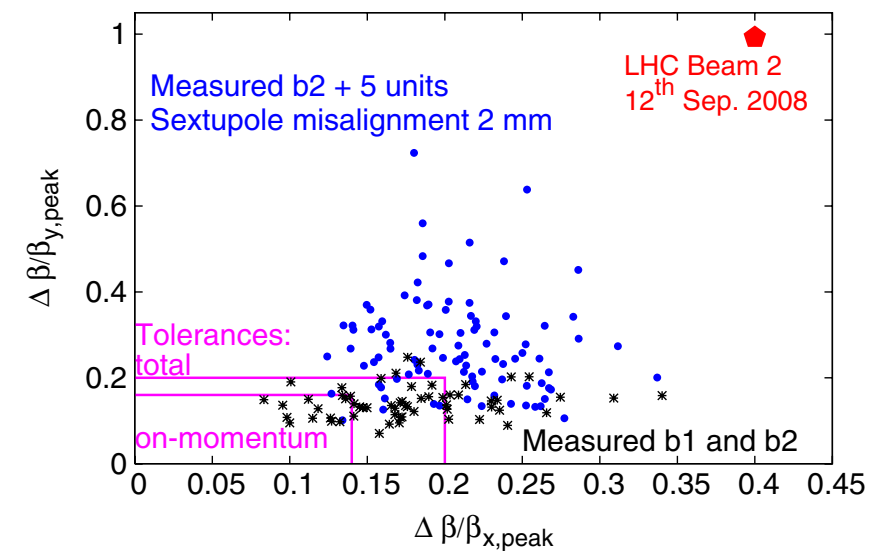

FIG. 5. (Color) Simulations (black and blue), observation (red), and tolerances (magenta) of the peak beta beating in the LHC, showing an unexpectedly large measured beta beating for beam 2 .

same qualitative information as the $\beta_{x, y}$, having both measurements turns out especially useful in finding gradient errors as explained in the next section. The beta beating is the relative deviation of the measured betas from the design betas. Figures 4 and 5 compare the LHC beam 2 reconstructed beta beating to the tolerances as presented in Ref. [1] and to simulations with realistic errors. The horizontal beta beating is within expected values for a first measurement and not far from tolerances. However, the large vertical beta beating can neither be explained by the measured multipolar components of the superconducting magnets nor by the observed closed orbit distortion [16]. This suggests that important optical errors might exist. New algorithms have been developed in order to identify possible gradient errors in this regime of large beta beating.

\section{OPTICS ERRORS RECONSTRUCTION}

A typical approach for optics correction in accelerators uses the inverse model response matrix of gradient strengths on some observables like phase advances, beta functions, or dispersion, see e.g. [3,20-23]. This is illustrated by the following equation:

$$
\left(\begin{array}{c}
\Delta \phi_{1} \\
\Delta \phi_{2} \\
\Delta \phi_{3} \\
\vdots \\
\Delta \phi_{N}
\end{array}\right)=\mathbf{R} \times\left(\begin{array}{c}
\Delta K_{1} \\
\Delta K_{2} \\
\Delta K_{3} \\
\vdots \\
\Delta K_{M}
\end{array}\right)
$$

where $\mathbf{R}$ is a rectangular matrix,

$$
\mathbf{R}=\left(\begin{array}{ccccc}
R_{11} & R_{12} & R_{13} & \cdots & R_{1 M} \\
R_{21} & R_{22} & R_{23} & \cdots & R_{2 M} \\
R_{31} & R_{32} & R_{33} & \cdots & R_{3 M} \\
\vdots & \vdots & \vdots & \ddots & \vdots \\
R_{N 1} & R_{N 2} & R_{N 3} & \cdots & R_{N M}
\end{array}\right),
$$


the $\phi_{i}$ s represent the $N$ phase advances between BPMs, while the $K_{i}$ s correspond to the $M$ gradient variables and $\mathbf{R}$ is the model response matrix that connects them. However, this approach is not directly suitable for this exceptional situation where the size of the errors exceeds the linear regime and there is no possibility to iterate corrections on the machine. Hence, a more local approach aiming at identifying errors has been developed.

\section{A. The segment-by-segment approach}

The entire machine is split into several segments and each of these segments is treated as an independent transfer line. The measured alpha and beta functions at the entrance of the segment are used as initial conditions for the optical parameters of the respective segment. The propagation of the initial conditions using the ideal model should follow the measurement as long as there are no gradient errors. Therefore, any deviation from the measured optics is easily identified by a direct comparison, thus localizing the gradient error. The amplitude of the errors within the segment can be determined by matching the propagated optics to the measured one via the preferred matching algorithm. The advantage of having a segmented machine is the considerable reduction of the dimensionality of the problem. Therefore, only the matching variables within the segment are used to reconstruct the measured optics. Following the illustration of Eq. (2), by having changed the reference model in every segment we have transformed the $\mathbf{R}$ matrix into a block diagonal matrix, with $n$ independent blocks, represented by the independent response matrices $\mathbf{R}_{\mathbf{i}}$,

$$
\mathbf{R}=\left(\begin{array}{ccccc}
\mathbf{R}_{1} & \mathbf{0} & \mathbf{0} & \cdots & \mathbf{0} \\
\mathbf{0} & \mathbf{R}_{2} & \mathbf{0} & \cdots & \mathbf{0} \\
\mathbf{0} & \mathbf{0} & \mathbf{R}_{3} & \cdots & \mathbf{0} \\
\vdots & \vdots & \vdots & \ddots & \vdots \\
\mathbf{0} & \mathbf{0} & \mathbf{0} & \cdots & \mathbf{R}_{\mathbf{n}}
\end{array}\right)
$$

This method naturally applies to LHC by splitting the ring into insertion regions (IRs) and arcs (see Ref. [24] for the details concerning the LHC layout). It is important to verify that the measured betas and alphas used as initial conditions for the propagated optics have small measurement errors.

This method proved most useful for IR3. This insertion houses the momentum cleaning systems of both beams [24]. Particles with a large momentum offset (typically larger than $1 \times 10^{-3}$ ) are scattered by the primary jaw of collimators in IR3 and are then absorbed by secondary collimators. This prevents the generation of beam losses in the superconducting arcs. The insertion optics is generated by 20 quadrupoles (which are shown in the top plot of Fig. 6). The central part of the insertion, where the

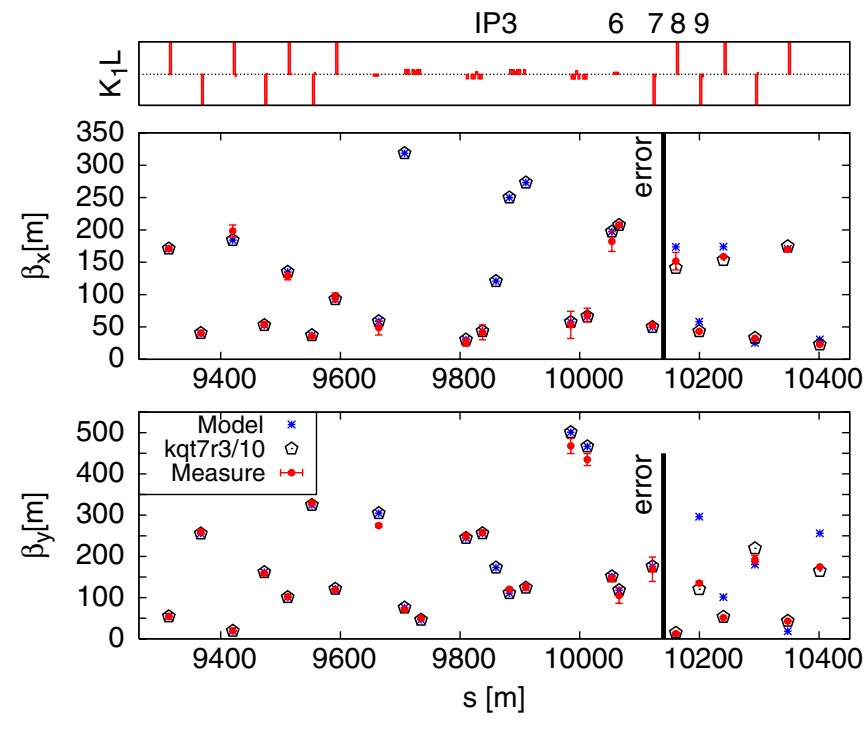

FIG. 6. (Color) Segment-by-segment approach in IR3. The top plot shows the gradient distribution versus longitudinal location. The two bottom plots show the horizontal and vertical beta functions from measurement and for two models (propagated by taking the initial $\alpha$ and $\beta$ as measured).

collimators are installed, features normal conducting magnets. The quadrupoles starting from Q6 up to the end of the dispersion suppressor are superconducting devices.

The two bottom plots of Fig. 6 show the horizontal and vertical beta functions from measurement and for two models (propagated by taking the initial $\alpha$ and $\beta$ as measured). The ideal model is represented by the blue stars which shows an excellent agreement with the measurement until the location $10200 \mathrm{~m}$, from which point onwards the vertical beta functions largely differ. This suggests that a gradient error exists between the 6th and the 9th quadrupoles (as indexed on the top plot). To restore the good agreement between model and measurement, the quadrupole mqtli7r3b2 had to be switched off or reduced by a factor of 10 (black pentagons on the figure), clearly suggesting some hardware problem with this quadrupole. This same feature was also observed in dispersion measurements [25]. The most likely hypothesis to explain this observation was a cable swap between the magnets of the two beams, namely mqtli7r3b2 and mqtli7r3b1. It was later found that this hypothesis was supported by previous hardware tests, which had indicated a possible voltage inversion between the corresponding voltage taps [26]. However, the results from these new analyses were crucial to determine that the voltmeters were actually properly connected and confirm the cable swap between magnets.

The segment-by-segment method is also applied to other sectors. Table II summarizes the current status of the findings. The error found in IR2 is significantly weaker than that of IR3. A $14 \%$ increase in the strength of one module of the fourth magnet on the left of IP2 would partly explain the observed local beta beating. This might be explained by 
TABLE II. Segment-by-segment summary. The second and third columns show the impact of the error on the ideal lattice. The first and fourth columns show the segment and the "best fit" corrector. Only the IR3 error has been confirmed as a real error.

\begin{tabular}{lccc}
\hline \hline Segment & $\begin{array}{c}\left(\frac{\Delta \beta_{x}}{\beta_{x}}\right)_{\text {peak }} \\
{[\%]}\end{array}$ & $\begin{array}{c}\left(\frac{\Delta \beta_{y}}{\beta_{y}}\right)_{\text {peak }} \\
{[\%]}\end{array}$ & Source (change) \\
\hline IR3 & 17 & 54 & mqtli7r3b2 $(/ 10)$ \\
IR2 & 9 & 5 & mqya412b2 $(+14 \%)$ \\
IR7 & 6 & 6 & mqt5[rl]7 $(\times[-2,3])$ \\
IR6 & 5 & 4 & mq416b2 $(+1 \%)$ \\
Arc 2-3 & 0 & 3 & $\operatorname{mqd23~}(+0.4 \%)$ \\
\hline \hline
\end{tabular}

a cable swap between beam 1 and beam 2 magnets. However, other errors distributed within the IR2 quadrupoles can also explain the observed beta beating. Hardware tests are currently being done to address this observation.

The fifth quadrupoles right and left of IP7 need to be scaled by factors -2 and 3 to reproduce the observed beta beating. These elements are powered at $0.9 \mathrm{~A}$, while the magnetic measurements were limited to $30 \mathrm{~A}$. Hence, a large uncertainty at very low field is not discarded. However, again this error is not the unique possibility to explain the observed beta beating.

A $1 \%$ increase in the strength of the fourth quadrupole left to IP6 has been assigned by the segment-by-segment method. This error is large when compared to the expected uncertainty of the transfer function. Therefore, this is just an effective error to reproduce the measured optics.

Finally, the segment-by-segment approach has assigned a systematic $0.4 \%$ error in the transfer function of the arc 2-3 defocusing quadrupoles. Other errors like sextupole misalignments or multipolar components could also explain or contribute to the observed beta beating.
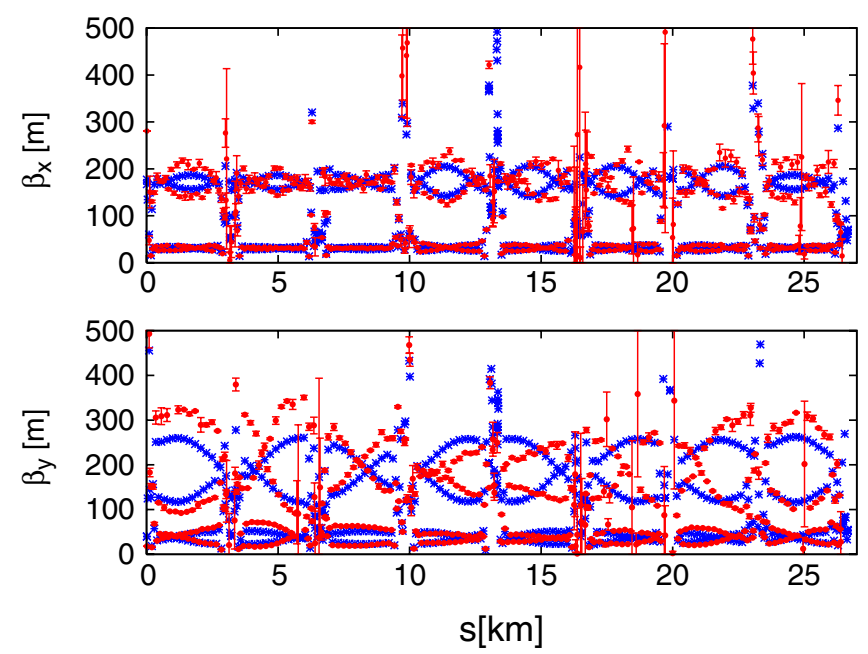

FIG. 7. (Color) Comparison of measured beta functions (red) and the model (blue) after including all the findings from the segment by segment.
It is clear that the error found in IR3 is the most important producing by itself more than $50 \%$ peak beta beating in the vertical plane. The rest of the errors can only be considered as a "best fit" and need further investigation. Figure 7 compares the model after including all the findings from the segment-by-segment approach to the measurement. The agreement is still not fully satisfactory in the vertical plane most likely due to existence of other, probably smaller, errors.

\section{B. The model iterative correction}

Yet another new method has been implemented to achieve the best possible optics error reconstruction. This method is based on the already mentioned inverse model response matrix (using the periodic optics), Eq. (2), but with an important new feature to allow for iterations. After each iteration the change in the model phase advance (obtained by introducing the corrections in the ideal model) is subtracted from the measured phase advances as a means to simulate an iterative correction in the real machine. It is worth mentioning that any correction algorithm could be used during iterations.

To obtain the best possible results, we have applied this new model iterative correction starting from a model already including the optical errors from the segment-bysegment approach. After five iterations a very satisfactory agreement is found between model and measurement, Fig. 8. The qualitative summary of the absolute values of the correction strengths integrated over the IR side is shown in Fig. 9.

It is reassuring to observe that the IR3 error remains the strongest and that no other relevant error has been found in IR3 by the iterative approach. However, IR2 needs further investigation, since the iterative correction finds comparable errors to those suggested by the segment-by-segment
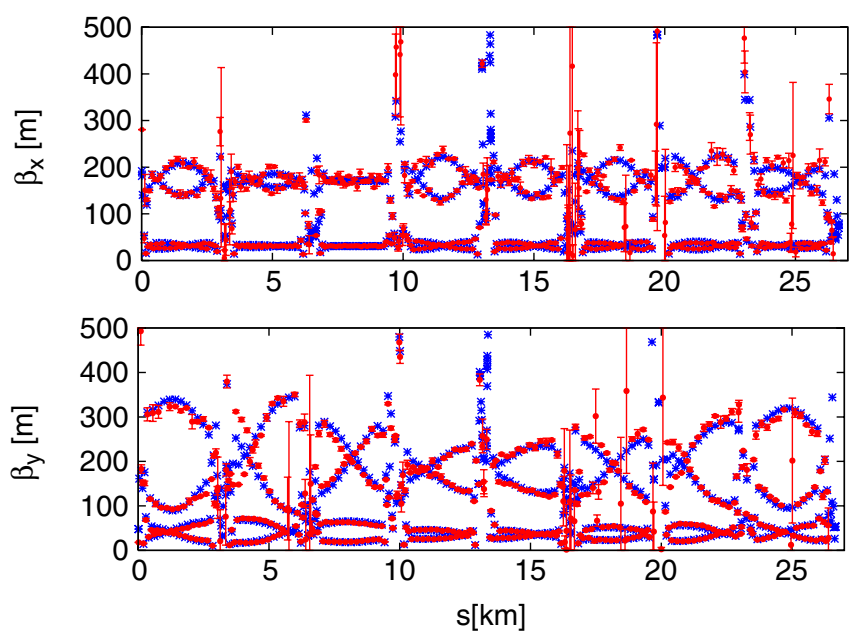

FIG. 8. (Color) Comparison of model (blue) and measured beta functions (red) after five iterations of the model iterative correction (having started from the segment-by-segment results). 


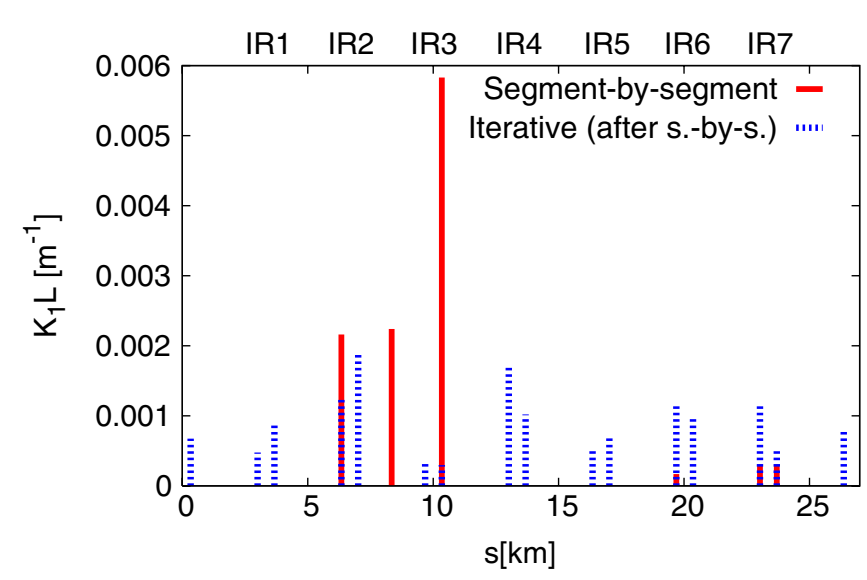

FIG. 9. (Color) Integrated error distribution as obtained by the segment-by-segment and the iterative approaches.

method. Figure 9 also reveals that IR1, IR5, and IR8 have rather small optics errors.

\section{COUPLING MEASUREMENT}

The linear coupling parameters are inferred from the secondary spectral lines, i.e., the vertical tune in the horizontal signal and vice versa; see, e.g., Refs. [27,28]. The difference coupling resonance driving term $f_{1001}$ is given by the following expression as a first order approximation on the coupling sources:

$$
f_{1001}(s)=\frac{\sum_{j} K_{j} \sqrt{\beta_{x, j} \beta_{y, j}} e^{i\left(\phi_{x, j}^{s}-\phi_{y, j}^{s}\right)}}{4\left(1-e^{2 \pi i\left(Q_{x}-Q_{y}\right)}\right)},
$$

where $K_{j}$ are the integrated coupling strengths, $\beta_{z, j}$ are the beta functions at the sources, and $\phi_{z, j}^{s}$ are the phase ad-
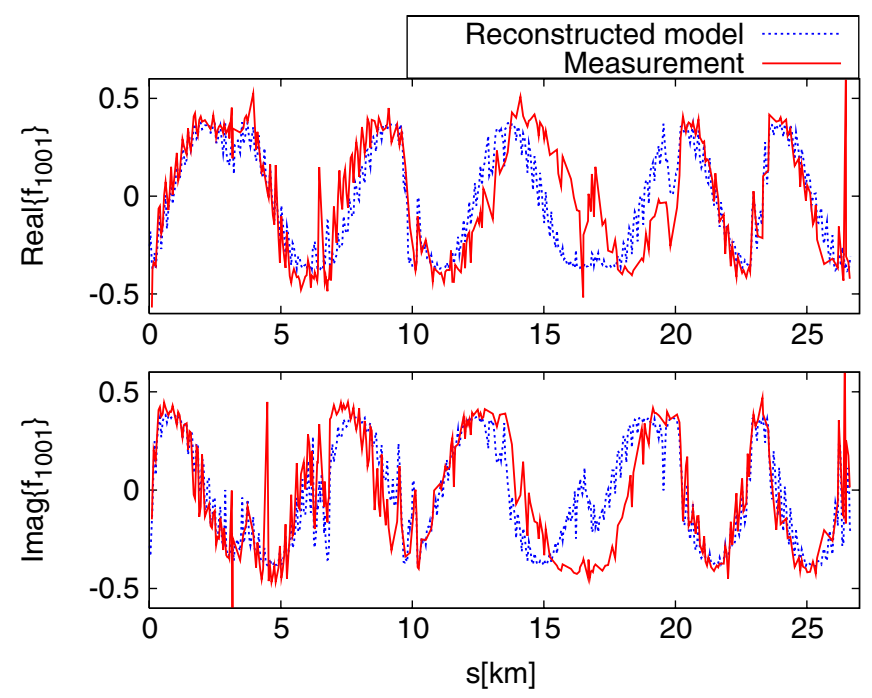

FIG. 10. (Color) Real and imaginary parts of the coupling resonance driving term $f_{1001}$ compared with a model reconstructed via the skew quadrupole response matrix. vances at the sources expanding the lattice from the evaluation point $s$, i.e., for a coupling source $j_{0}$ at $s_{0}, \phi_{z, j_{0}}^{s_{0}}=0$.

Figure 10 compares the measured real and imaginary parts of the difference coupling resonance driving term $f_{1001}$ with a reconstructed model. This reconstruction is based on the response matrix of the machine skew quadrupole correctors. The five periods observed in the oscillations of the real part of $f_{1001}$ correspond exactly to the integer tune split between the horizontal and vertical tunes, thus experimentally confirming that the machine had the same integer tune split as the model.

Linear coupling is generated by the rotation of quadrupole magnets, a nonzero vertical closed orbit at the sextupoles, and the $a_{2}$ magnetic errors in the superconducting dipoles. There were no skew quadrupole correctors excited during the measurement. Repeating this measurement with better quality data should give an insight into the sources of coupling guided by theory [27] and simulations [3].

\section{CONCLUSIONS AND OUTLOOK}

The only available turn-by-turn BPM data acquisition, at injection, has served to test and compare various methods to measure the periodic ring optics for the counterclockwise rotating beam. In the constrained circumstances with only 90 turns, uncorrected coupling and large chromaticity, the SVD approach proved to be the most accurate. The measured vertical beta beating is found to be larger than predicted by the specified field and alignment errors, suggesting that a few additional large error sources may exist. Two new methods to address the localization of optics correction were developed, namely segment-by-segment and model iterative correction. The application of the segment-by-segment approach to IR3 led to the identification of a dominant optics error for the counterclockwise beam. Evidence from previous hardware tests supported the hypothesis that this error was caused by a cable swapping between the beam 2 and beam 1 magnets mqtli7r3b2 and mqtli7r3b1. Using the model iterative correction, the optics has been very well reconstructed by using distributed sources all around the ring, confirming that there are no more relevant errors in IR3, IR1, IR5, and IR8. Error sources in IR2 still remain unclear and further investigations are needed.

\section{ACKNOWLEDGMENTS}

These data only exist due to the exceptional work of the LHC operation and instrumentation teams. We thank O. Brüning for the supervision of this work. We are thankful to E. Calvo Giraldo and R. Jones for providing feedback on the BPM status. We thank N. Catalán Lasheras for providing the critical information concerning the results of the hardware tests of mqtli7r3b2. We also thank Y. Papaphilippou, S. Peggs, S. Redaelli, F. Schmidt, and R. Steinhagen for the very useful discussions. 


\section{APPENDIX: SIMULATION OF MEASUREMENT RESOLUTION AND ACCURACY}

In order to fully understand the random and systematic errors of the beta-beating measurement, numerical simulations have been performed using the reconstructed model including normal and skew quadrupolar errors. The ingredients of the simulations are as follow: (i) A single onmomentum particle is tracked for 90 turns using the reconstructed optics, while recording the horizontal and vertical positions at all the BPMs. (ii) The initial conditions are set to reproduce the measured horizontal and vertical oscillation amplitudes: $A_{x} \approx 2.0 \sigma$ and $A_{y} \approx 3.8 \sigma$, where $\sigma$ is the design beam size at injection, $\sigma=\sqrt{\epsilon \beta}$ with $\epsilon=$ $7.8 \times 10^{-9} \mathrm{~m}$. (iii) A Gaussian random error with $0.2 \mathrm{~mm}$ standard deviation is added to the tracked positions. This error represents the BPM resolution and is consistent with specifications and direct measurements [13,29,30]. (iv) The same analysis as applied to the experimental data is used in the simulations. Figure 11 shows the histograms of the relative beta function error from simulation for SVD and Sussix algorithms. This figure is to be compared with Fig. 2. The agreement between measurement and simulation confirms the expected quality of the BPM data and that the large difference in the SVD and Sussix performance is strictly due to the discrimination of uncorrelated noise in the SVD algorithm.

The systematic error of the measurement can be estimated by computing the relative deviation of the simulated measured betas and the betas from the model as used in the simulation. Obviously, this can only be done in simulation. Figure 12 shows the histogram of this systematic relative error as obtained from the simulation. Via Gaussian fits, the rms errors are estimated to be 5\% and 3\% for the horizontal
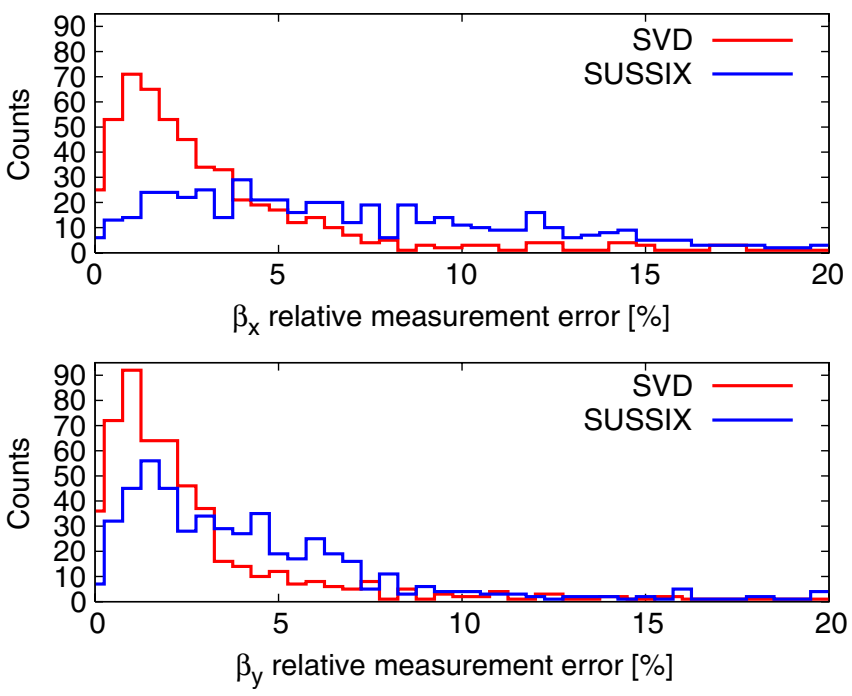

FIG. 11. (Color) Simulation of the performance of two different algorithms used to measure the $\beta$ functions. Histograms of the horizontal and vertical beta function relative measurement errors are shown on the top and bottom plots, respectively.
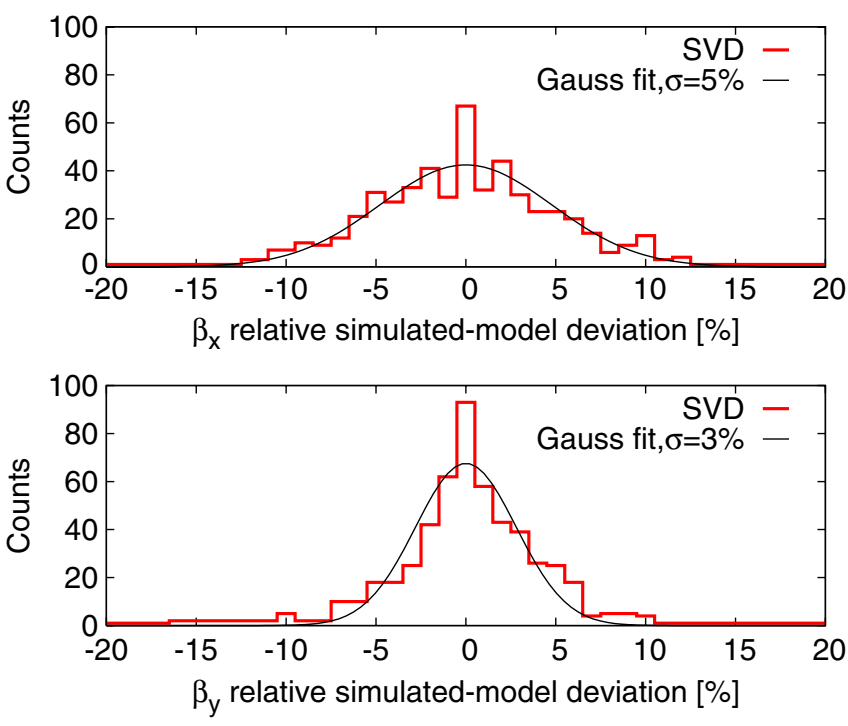

FIG. 12. (Color) Relative deviation of measured and model beta functions as obtained from simulation.

and vertical planes, respectively. These systematic errors are slightly larger than the estimated error bars of $4 \%$ and $2 \%$ but still small confirming the robustness of the betabeating measurement. The systematic errors will be reduced in future measurements by increasing the number of turns and improving the machine conditions.

[1] S. Fartoukh and O. Brüning, CERN-LHC-Project-Report501, 2001 (internal publication).

[2] F. Zimmermann, S. Fartoukh, and R.W. Assmann, in Proceedings of the Eighth European Particle Accelerator Conference, edited by J. Poole and C. PetitJean-Genaz (Institute of Physics, London, 2002), p. 296.

[3] R. Tomás, O. Brüning, R. Calaga, S. Fartoukh, A. Franchi, M. Giovannozzi, Y. Papaphilippou, S. Peggs, and F. Zimmermann, in Proceedings of the Tenth European Particle Accelerator Conference, edited by J. Poole and C. Petit-Jean-Genaz (Institute of Physics, London, 2006), p. 2023.

[4] R. Calaga, R. Tomás, and F. Zimmermann, in 2007 Particle Accelerator Conference Proceedings, edited by C. Petit-Jean-Genaz (Institute of Physics, London, 2007), p. 3693.

[5] M. Aiba, R. Calaga, A. Morita, R. Tomás, and G. Vanbavinckhove, in Proceedings of the Eleventh European Particle Accelerator Conference Proceedings, edited by C. Petit-Jean-Genaz (Institute of Physics, London, 2008), p. 2572.

[6] P. Hagen, M. Giovannozzi, J.-P. Koutchouk, T. Risselada, S. Sanfilippo, E. Todesco, and E. Wildner, in Proceedings of the Tenth European Particle Accelerator Conference (Ref. [3]), p. 2248.

[7] P. Hagen, M. Giovannozzi, J.-P. Koutchouk, T. Risselada, F. Schmidt, E. Todesco, and E. Wildner, in Proceedings of 
the Eleventh European Particle Accelerator Conference Proceedings (Ref. [5]), p. 1744.

[8] O. Aberle et al., CERN LHC-Performance-Note-001, 2008 (internal publication).

[9] L. Evans, "LHC Commissioning \& Status," in the 9th ICFA Seminar, http://www-conf.slac.stanford.edu/ icfa2008/.

[10] Measurements performed during the 24th of August of 2008.

[11] J. Wenninger, "YASP: Yet Another Steering Program."

[12] A. Boccardi, M. Gasior, O. R. Jones, P. Karlsson, and R. J. Steinhagen, CERN LHC-Performance-Note-007, 2009 (internal publication).

[13] M. Aiba, R. Calaga, A. Franchi, M. Giovannozzi, V. Kain, A. Morita, R. Tomás, G. Vanbavinckhove, and J. Wenninger, CERN LHC-Performance-Note-008, 2009 (internal publication).

[14] P. Castro-Garcia, Ph.D. thesis, University of Valencia, Spain [CERN-SL-96-70-BI, 1996].

[15] R. Calaga and R. Tomás, Phys. Rev. ST Accel. Beams 7, 042801 (2004).

[16] M. Aiba et al., in Proceedings of the 2009 Chamonix Workshop on LHC Performance (CERN-ATS-2009-001, 2009), p. 205.

[17] J. Irwin, C. X. Wang, Y. T. Yan, K. L. F. Bane, Y. Cai, F.-J. Decker, M. G. Minty, G. V. Stupakov, and F. Zimmermann, Phys. Rev. Lett. 82, 1684 (1999).

[18] R. Calaga, Ph.D. thesis, Stony Brook University, 2006.
[19] R. Bartolini and F. Schmidt, CERN SL-Note-98-017-AP, 1998 (internal publication).

[20] A. Morita, H. Koiso, Y. Ohnishi, and K. Oide, Phys. Rev. ST Accel. Beams 10, 072801 (2007).

[21] D. Sagan, R. Meller, R. Littauer, and D. Rubin, Phys. Rev. ST Accel. Beams 3, 092801 (2000).

[22] M. Minty and F. Zimmermann, Measurement and Control of Charged Particle Beams (Springer, Berlin, 2003).

[23] G. Arduini, F. Zimmermann, and C. Carli, in Proceedings of the 9th European Particle Accelerator Conference, Lucerne, 2004 (EPS-AG, Lucerne, 2004) (CERN-AB2004-083).

[24] O. Brüning, P. Collier, Ph. Lebrun, S. Myers, R. Ostojic, J. Poole, and P. Proudlock, CERN-2004-003-V-1, 2004.

[25] M. Lamont, CERN LHC-Performance-Note-009, 2009 (internal publication).

[26] N. Catalán Lasheras (private communication).

[27] R. Calaga, R. Tomás, and A. Franchi, Phys. Rev. ST Accel. Beams 8, 034001 (2005).

[28] R. Tomás, Ph.D. thesis, University of Valencia, Spain, 2003.

[29] J.-P. Koutchouk, CERN LHC-BPM-ES-0004 rev2.0, 2002 (internal publication).

[30] K. Fuchsberger, V. Kain, M. Lamont, L. Ponce, W. Venturini-Delsolaro, J. Wenninger, A. Butterworth, S. Fartoukh, R. Tomás, and F. Zimmermann, CERN LHCPerformance-Note-002, 2008 (internal publication). 